TRANSACTIONS OF THE

AMERICAN MATHEMATICAL SOCIETY

Volume 178, April 1973

\title{
THE TRACE-CLASS OF A FULL HILBERT ALGEBRA
}

BY

\author{
MICHAEL R. W. KERVIN( $\left.{ }^{1}\right)$
}

\begin{abstract}
The trace-class of a full Hilbert algebra $A$ is the set $\tau(A)=$ $\{x y \mid x \in A, y \in A\}$. This set is shown to be a $*$-ideal of $A$, and possesses a norm $\tau$ defined in terms of a positive hermitian linear functional on $\tau(A)$. The norm $\tau$ is in general both incomplete and not an algebra norm, and is also not comparable with the Hilbert space norm \|\| on $\tau(A)$. However, a one-sided ideal of $\pi(A)$ is closed with respect to one norm if and only if it is closed with respect to the other. The topological dual of $\tau(A)$ with respect to the norm $\tau$ is isometrically isomorphic to the set of left centralizers on $A$.
\end{abstract}

Introduction. The methods of Schatten [10], employed by Saworotnow and Friedell [8] in the $H^{*}$-algebra setting, are used here in $\$ \$ 1$ and 2 , to show that the trace-class $r(A)$ of a full Hilbert algebra $A$ is a *ideal of $A$ (Theorem 2.2), and to define a norm $\tau$ and a positive hermitian linear functional on $\tau(A)$. These enjoy many of the same properties as for $H^{*}$-algebras, with some exceptions (Theorem 2.5): the norm $\tau$ is generally incomplete and is not an algebra norm on $\tau(A)$, unless $A$ itself is complete, in which case $A$ is an $H^{*}$-algebra in a trivially equivalent norm.

$\$ 3$ deals with two theorems concerning the trace-class (see [10, IV.1 $_{\text {, }}$, and also [9] for the $H^{*}$-algebra setting). Theorem 3.1 shows that the topological dual of $x(A)$ is isometrically isomorphic to the set of left centralizers on $A$, while Theorem 3.2 says that $r(A)$ is isometrically isomorphic to a subspace of $C^{*}(A)$, the $C^{*}$-algebra of $A$. An example is given (3.3) to show that this subspace may not even be dense in $C^{*}(A)$.

In $\$ 4$ we examine the relation between the two norms $\tau$ and \|\| on the traceclass. They are in general incomparable (Theorem 2.5 and 4.1). However, in Theorem 4.5 it is shown that a one-sided ideal of $\pi(A)$ is closed in one norm topology if and only if it is closed in the other. It is noted (Theorem 4.7) that $x(A)$ is an orthocomplemented Hilbert algebra. The final result is that the closed ideals of $r(A)$ are precisely the trace-classes of closed ideals of $A$. Many of these results

Presented to the Society, March 27, 1972; received by the editors March 24, 1972.

AMS (MOS) subject classifications (1970). Primary 46K15; Secondary 46K05.

Key words and phrases. Full Hilbert algebra, projection base, left (right) centralizer, trace-class, orthogonal complement.

(1) Most of this paper is taken from the author's doctoral dissertation written under the supervision of Professor B. Yood at the University of Oregon. The author wishes to express his appreciation to Professor Yood for his guidance and encouragement. 
are generalizations of the same occurrences for $H^{*}$-algebras, as discovered by Smith in [11].

1. Basic results. Let $A$ denote a Hilbert algebra with inner product $(x, y)$, norm $\|x\|=(x, x)^{1 / 2}$, and involution $x \rightarrow x^{*}$. The definition and elementary properties of a Hilbert algebra can be found in [1] and [7]; see also [2], [6] and [12]. Our notation follows that of Yood in [14]. The Hilbert space which is the completion of $A$ in the norm $\|x\|$ is denoted $H$, and $A_{b}$ is the fulfillment of $A . B(H)$ shall denote the space of all bounded linear operators on $H$.

Put $\Lambda(A)=\left\{\bar{R}_{y}: y \in A\right\}^{\prime}$ (the commutant taken in $\mathcal{B}(H)$ ) and $P(A)=\left\{\bar{L}_{x}: x \in A\right\}^{\prime}$. The commutation theorem (see [2] or [7]) states that $\Lambda(A)^{\prime}=P(A)$ and $P(A)^{\prime}=$ $\Lambda(A)$. Moreover, $\Lambda(A)$ (resp. $P(A)$ ) is the strong closure of $\left\{\bar{L}_{x}: x \in A\right\}$ (resp. of $\left.\left\{\bar{R}_{y}: y \in A\right\}\right)$, and $\Lambda(A)=\Lambda\left(A_{b}\right), P(A)=P\left(A_{b}\right)$. Since $A_{b}$ is invariant under $\Lambda\left(A_{b}\right) \cup P\left(A_{b}\right)$ [7, Proposition 1.6], $\Lambda\left(A_{b}\right)$ may also be defined as the set of operators in $\mathfrak{B}(H)$ satisfying $T(x y)=T(x) y, \forall x$ and $y$ in $A_{b}$. We call such operators left centralizers on $A_{b}$ after B. E. Johnson [4]; operators in $P\left(A_{b}\right)$ are called right centralizers on $A_{b}$. Johnson's terminology differs (i.e. "left" in place of "right") from other notions of centralizer found, for example, in [13] and [5].

As in [8], a projection shall be a self-adjoint idempotent, and a projection base for $A$ shall be a maximal family of nonzero mutually orthogonal projections of $A$. A Hilbert algebra in general need not contain any nonzero projections (see $[14, \S 4]$ ), but projection bases exist in full Hilbert algebras (see [7, Theorem 2.3]). Thus we restrict our attention to the latter; in the remainder of the paper, $A$ shall denote a full Hilbert algebra.

We need two results of Rieffel [7, Theorems 3.8 and 3.9], but enlarged to involve projection bases.

1.1 Lemma. Let $T \in \Lambda(A)$ (resp. $T \in P(A)$ ). The following statements are equivalent:

(1) $T=\bar{L}_{x}$ (resp. $T=\bar{R}_{x}$ ) for some $x$ in $A$.

(2) $\sup \{\|T e\|: e$ is a projection of $A\}<+\infty$.

(3) There is a projection base $\left\{e_{\gamma}: \gamma \in \Gamma\right\}$ for $A$ such that $\Sigma_{\gamma \in \Gamma}\left\|T e_{\gamma}\right\|^{2}<+\infty$.

1.2 Lemma. Let $a \in A$. The following statements are equivalent:

(1) $a$ is positive and integrable.

(2) $a$ is positive and $\Sigma_{\gamma \in \Gamma}\left(a, e_{\gamma}\right)<+\infty$ for some projection base $\left\{e_{\gamma}: \gamma \in \Gamma\right\}$ for $A$.

(3) $a=b^{2}$ for some unique positive $b$ in $A$.

(4) $a=x^{*} x$ for some $x$ in $A$. 
Proof. Clearly (3) $\Rightarrow(4) \Rightarrow(1) \Rightarrow(2)$. If (2) holds, and $T$ is the unique positive square root in $\Lambda(A)$ of the positive operator $\bar{L}_{a}$, then

$$
\sum_{\gamma \in \boldsymbol{\Gamma}}\left\|T e_{\gamma}\right\|^{2}=\sum_{\gamma \in \boldsymbol{\Gamma}}\left(T^{2} e_{\gamma}, e_{\gamma}\right)=\sum_{\gamma \in \boldsymbol{\Gamma}}\left(a e_{\gamma}, e_{\gamma}\right)<+\infty
$$

so $T=\bar{L}_{b}$ for some positive $b$ in $A$ by Lemma 1.1. Since the mapping $x \rightarrow \bar{L}_{x}$ is an algebra ${ }^{*}$-isomorphism on $A$, it follows that $b$ is the unique positive square root of $a$. Thus (2) $\Rightarrow(3)$.

Using notation of Schatten (see [10] and [8]), we let $[x]$ denote the positive square root in $A$ of $x^{*} x$, for each $x$ in $A$. Note that $\|[x]\|=\|x\|$, and $\bar{L}_{[x]}=$ $\left[\bar{L}_{x}\right]$, the positive square root of $\bar{L}_{x}{ }^{*}$.

It is interesting to observe that a complete analogue of the polar decomposition theorem for operators in $\mathcal{B}(H)$ (see [10]) obtains in the full Hilbert algebra setting. The partial isometry involved does not have as exact a description as for $\mathfrak{B}(H)$ or any $H^{*}$-algebra (see [8]), however that is an unnecessary detail.

1.3 Theorem. For each $x$ in $A$, there is a partial isometry $W_{x}$ in $\Lambda(A)$ with initial set $\overline{[x] A}^{H}$ (the closure in $H$ of $[x] A$ ) and final set $\overline{x A}^{H}$ such that

(1) $x=W_{x}([x])$;

(2) $[x]=W_{x}^{*}(x)$;

(3) $x^{*}=W_{x}^{*}\left(\left[x^{*}\right]\right)$;

(4) $\left[x^{*}\right]=W_{x}\left(x^{*}\right)$.

Moreover, if $x=W(b)$ for some positive $b$ in $A$ and partial isometry $W$ in $\Lambda(A)$ with initial set $\overline{b A}^{H}$, then $b=[x]$ and $W=W_{x}$.

Proof. Use the polar decomposition theorem in [10] to obtain a partial isometry $W_{x}$ in $\mathfrak{B}(H)$ with initial set $\overline{[x] A}^{H}$ and final set $\overline{x A}^{H}$ such that $\bar{L}_{x}=W_{x}\left[\bar{L}_{x}\right]=$ $W_{x} \bar{L}_{[x]}$, etc. For convenience let $\pi$ denote the orthogonal complement in $H$ of $\overline{[x] A}^{H}$. $\pi$ is invariant under each $\bar{R}_{y}(y$ in $A)$ so $\bar{R}_{y} W_{x}$ and $w_{x} \bar{R}_{y}$ agree on $H=\overline{[x] A}^{H} \oplus \pi$, for each $y$ in $A$. Thus $W_{x}$ is a left centralizer. (1)-(4) now follow using the semisimplicity of $A$.

If $x=W(b)$ as in the last sentence of the theorem, then

$$
\bar{L}_{x^{*} x}=\bar{L}_{x}^{*} \bar{L}_{x}=\bar{L}_{b} W{ }^{*} W \bar{L}_{b}=\bar{L}_{b}^{2}=\bar{L}_{b 2},
$$

so $b=[x]$. If follows that $W$ and $W_{x}$ agree on $\overline{[x] A}$, hence are equal.

As one might expect, there is a parallel result concerning right centralizers.

1.4 Theorem. For each $x$ in $A$, there is a partial isometry $V_{x}$ in $P(A)$ with initial set $\overline{A[x]}^{H}$ and final set $\overline{A x}^{*} H$, such that

(1) $x^{*}=V_{x}([x])$

(2) $[x]=V_{x}^{*}\left(x^{*}\right)$; 
(3) $x=V_{x}^{*}\left(\left[x^{*}\right]\right)$;

(4) $\left[x^{*}\right]=V_{x}(x)$.

If $x^{*}=V(b)$ for some positive $b$ in $A$ and partial isometry $V$ in $P(A)$ with initial set $\overline{A b}^{H}$, then $b=[x]$ and $V=V_{x}$.

Suppose for any $S$ in $\mathcal{B}(H)$ we define an operator $S^{\#}$ on $H$ by $S^{\#}(\xi)=S\left(\xi^{*}\right)^{*}$, $\xi$ in $H$. The mapping $S \rightarrow S^{\#}$ is a conjugate-linear isometric automorphism of period 2 of $\mathcal{B}(H)$ onto itself, with the following properites:

(a) $\bar{L}_{x}^{\#}=\bar{R}_{x}{ }^{*}$, for any $x$ in $A[2$, Lemma 3$]$;

(b) $\Lambda(A)^{\#} \stackrel{x}{=} P(A), P(A)^{\#}=\Lambda(A)$;

(c) if $P_{\pi}$ is the projection of $H$ onto a closed subspace $M$, then $P_{\pi}^{\#}=P_{\pi^{*}}$;

(d) if $U$ is a partial isometry with initial set $\mathbb{N}$ and final set $\pi$, then $U^{\#}$ is a partial is ometry with initial set $\pi^{*}$, final set $\varkappa^{*}$;

(e) ${ }^{\#}$ commutes with ${ }^{*}$, the adjoint operation on $\mathcal{B}(H)$.

From this it follows that $V_{x}=W_{x}^{\#}, W_{x}{ }^{*}=W_{x}^{*}$, and $V_{x}{ }^{*}=V_{x}^{*}$ for each $x$ in $A$.

2. The trace-class. The trace-class of $A$ is the set $f(A)=\{x y: x \in A, y \in A\}$. This set is not obviously closed under addition. To show that this is so, we emulate the procedure in [10] and, more exactly, in [8]. To begin with, every element in the trace-class is integrable. It is not clear whether the converse obtains-however, it does for positive elements.

2.1 Lemma. For any $a$ in $A$, the following statements are equivalent:

(1) $a$ is in $r(A)$.

(2) $[a]$ is in $\tau(A)$.

(3) $[a]$ is integrable.

(4) There is a projection base $\left\{e_{\gamma}: y \in \Gamma\right\}$ for $A$ sucb that $\Sigma_{\gamma \in \Gamma}\left([a], e_{\gamma}\right)<+\infty$ o

(5) $[a]$ has a unique positive square root $[a]^{1 / 2}$ in $A$.

Proof. Use 1.2 and Theorem 1.3.

For any $x$ and $y$ in $A$ and any projection base $\left\{e_{y}: y \in \Gamma\right\}$ for $A$, the sum $\Sigma_{y \in \Gamma}\left(x y, e_{y}\right)$ converges absolutely to the number $\left(x, y^{*}\right)$, and is therefore independent of the choice of projection base. This number is called the trace of $x y$, $\operatorname{tr}(x y): \operatorname{tr}(a)=\Sigma_{\gamma \in \Gamma}\left(a, e_{\gamma}\right)$ for any $a$ in $\pi(A)$ and projection base $\left\{e_{y}: \gamma \in \Gamma\right\}$ for $A$.

2.2 Theorem. $r(A)$ is a dense *ideal of $A$ which is invariant under left or right centralizers. tr is a positive bermitian linear functional on $r(A)$ such that

(1) $\operatorname{tr}(x y)=\operatorname{tr}(y x)=\left(x, y^{*}\right)$,

(2) $\operatorname{tr}\left(x^{*} x\right)=\|x\|^{2}$,

for any $x$ and $y$ in $A$. 
Proof. It is clear that $\tau(A)$ is invariant under left or right centralizers. The proof that $r(A)$ is closed under addition is similar to Schatten's [10, Lemma 3 , p. 38]. The rest of the theorem now follows easily.

Now define $\gamma(a)=\operatorname{tr}([a])$ for each $a$ in $\gamma(A)$. Then $\gamma(a)=\Sigma_{\gamma \in \Gamma}\left([a], e_{\gamma}\right)$ for each projection base $\left\{e_{\gamma}: \gamma \in \Gamma\right\}$ for $A$. Right away we see that

$$
\tau(a)=\tau([a])=\left\|[a]^{1 / 2}\right\|^{2}
$$

and $\tau(\lambda a)=\operatorname{tr}(|\lambda|[a])=|\lambda| \tau(a)$, for any $a$ in $\gamma(A)$ and complex number $\lambda$. $\tau$ will be a norm on the trace-class once we show it is subadditive, and so we come to the next result (see [10, p. 39] as well as [8]).

2.3 Lemma. For any $a$ in $\tau(A)$ and operator $T$ in $\Lambda(A) \cup P(A)$,

(1) $|\operatorname{tr}(T a)| \leq\|T\| r(a)$,

(2) $r(T a) \leq\|T\| \tau(a)$.

Proof. If.$T$ is a left centralizer, the proof is the same as in [8]. If $T$ is a right centralizer, we proceed slightly differently:

$$
\begin{aligned}
|\operatorname{tr}(T a)| & =\left|\operatorname{tr}\left(T W_{a}\left([a]^{1 / 2}[a]^{1 / 2}\right)\right)\right|=\left|\operatorname{tr} T\left(W_{a}\left([a]^{1 / 2}\right)[a]^{1 / 2}\right)\right| \\
& =\left|\operatorname{tr} W_{a}\left([a]^{1 / 2}\right) T\left([a]^{1 / 2}\right)\right|=\left|\left(W_{a}[a]^{1 / 2},\left(T[a]^{1 / 2}\right) *\right)\right| \\
& \leq\left\|W_{a}[a]^{1 / 2}\right\|\left\|T[a]^{1 / 2}\right\| \leq\left\|[a]^{1 / 2}\right\|\|T\|\left\|[a]^{1 / 2}\right\|=\|T\| \tau(a) .
\end{aligned}
$$

Since $T$ commutes with operators in $\Lambda(A)$, we have

$$
\begin{aligned}
\tau(T a) & =\operatorname{tr}([T a])=\operatorname{tr}\left(W_{T a}{ }^{*}(T a)\right)=\operatorname{tr}\left(T W_{T a}{ }^{*}(a)\right) \\
& \leq\|T\| r\left(W_{T a}{ }^{*}(a)\right) \leq\|T\|\left\|W_{T a}{ }^{*}\right\| r(a) \leq\|T\| \tau(a) .
\end{aligned}
$$

2.4 Theorem. $\tau$ is a linear space norm on $\pi(A)$ with the following properties:

(1) multiplication in $x(A)$ is separately $r$-continuous;

(2) $x\left(a^{*}\right)=x(a)$ for each $a$ in $r(A)$;

(3) $|\operatorname{tr} a| \leq x(a)$ for each $a$ in $\pi(A)$;

(4) $\pi(x y) \leq\|x\|\|y\|$ for every $x$ and $y$ in $A$;

(5) $r(T)=\|T\|$ for every $T$ in $\Lambda(A) \cup P(A)$.

Proof. $\tau$ is subadditive: for any $a$ and $b$ in $\tau(A)$, we have

$$
\begin{aligned}
\tau(a+b) & =\operatorname{tr}([a+b])=\operatorname{tr}\left(W_{a+b}^{*}(a)+W_{a+b}^{*}(b)\right) \\
& \leq\left|\operatorname{tr}\left(W_{a+b}^{*}(a)\right)\right|+\left|\operatorname{tr}\left(W_{a+b}^{*}(b)\right)\right| \leq \tau(a)+\tau(b),
\end{aligned}
$$

using Theorem 1.3 and Lemma 2.3. Thus $\tau$ is a linear space norm on the traceclass. (1) follows from Lemma 2.3 also, as does (3). To prove (2), we have, using Theorem 1.4 and Lemma 2.3, 


$$
\tau\left(a^{*}\right)=\tau\left(V_{a}([a])\right) \leq\left\|V_{a}\right\| \tau([a])=\tau(a)
$$

for each $a$ in $\tau(A)$, so equality obtains. (4) is proven as in [8, Corollary 4]. If $T$ is a left or right centralizer on $A$, its restriction to the normed linear space $\tau(A)$ is continuous with respect to the norm $\tau$ by Lemma 2.3. The norm of the restricted operator is denoted $x(T)$ :

$$
\tau(T)=\sup \{\tau(T a): a \in \tau(A) \text { and } \tau(a) \leq 1\} .
$$

Now $r(T) \leq\|T\|$ by 2.3 . We prove the reverse inequality for left centralizers (proof is similar for right centralizers): for any $x$ in $A$,

$$
\begin{aligned}
\|T x\|^{2} & =\left\|(T x)^{*}\right\|^{2}=\tau\left(T x(T x)^{*}\right) \\
& =\tau\left(T\left(x(T x)^{*}\right)\right) \leq \tau(T)_{\tau}\left(x(T x)^{*}\right) \leq \tau(T)\|x\|\|T x\| \quad \text { by (4) above; }
\end{aligned}
$$

thus $\|T x\| \leq \tau(T)\|x\|$ for each $x$ in $A$. Thus $\|T\| \leq \tau(T)$, so equality obtains.

Thus far the trace-class $\gamma(A)$ and its norm $\tau$ have behaved much the same as in the $H^{*}$-algebra setting. Now however we notice some differences: $\tau$ is not an algebra norm on $\tau(A)$, and is incomplete. One may attribute these failings to the lack of the same properties of the norm \|\| on $A$, as we see from the next result.

2.5 Theorem. The following statements are equivalent:

(1) Multiplication in $r(A)$ is jointly $\tau$-continuous.

(2) There is a constant $M>0$ such that $\tau(a b) \leq M r(a) \tau(b)$ for every $a$ and $b$ in $r(A)$.

(3) There is a constant $K>0$ sucb that $\|a\| \leq K \pi(a)$ for eacb a in $r(A)$.

(4) $\tau$ is a complete norm on $\tau(A)$.

(5) \|\| is a complete norm on $A$ (so $A=H$ ).

(6) Multiplication in $A$ is jointly continuous.

(7) $A$ is trivially renormable to be an $H^{*}$-algebra.

Proof. (5), (6), and (7) are equivalent by Lemma 4.5 of [14]. The equivalence of (1) and (2) is a simple matter. If (2) is true, then for each $a$ in $t(A)$,

$$
\|a\|^{2}=\tau\left(a^{*} a\right) \leq M \tau\left(a^{*}\right)_{\tau}(a)=M \tau(a)^{2}
$$

so (3) holds. If (3) is true, then so is (6): for any $x$ and $y$ in $A$,

$$
\|x y\| \leq K \tau(x y) \leq K\|x\|\|y\| \quad \text { (using (4) of Theorem 2.4). }
$$

Suppose now that $A$ is trivially renormable to be an $H^{*}$-algebra, and suppose the $H^{*}$-algebra norm on $A$ is $\|x\|_{1}=c\|x\|(x$ in $A)$. Then $(x, y)_{1}=c^{2}(x, y)$ for all $x$ and $y$ in $A$, so $\tau_{1}(a)=c^{2} r(a)$ for all $a$ in $\pi(A)=\tau_{1}(A)$. But $\tau_{1}(A)=\gamma(A)$ is a Banach algebra in the norm $\tau_{1}$ (see [8] and [9]), hence $\tau$ itself is complete on the trace-class. Thus (7) $\Rightarrow(4)$. Finally, suppose (4) is true; then Lemma 2.3 implies that 


$$
\sup \left\{\tau\left(\bar{L}_{a}(b)\right): a \in \tau(A), \tau(a) \leq 1\right\} \leq\left\|\bar{R}_{b}\right\|<+\infty
$$

for each $b$ in $\tau(A)$. An application of the uniform boundedness principle gives

$$
M \equiv \sup \left\{\tau\left(\bar{L}_{a}\right): a \in \tau(A), \tau(a) \leq 1\right\}<+\infty .
$$

It follows that $\tau(a b)=\tau((a / \tau(a)) \cdot \tau(a) b) \leq M \tau(\tau(a) b)=M \tau(a) \tau(b)$ for all $a \neq 0$ in $\tau(A)$ and $b$ in $\tau(A)$; thus $(2)$ is true. This completes the proof of the theorem.

3. The dual of the trace-class. What follows now is an attempt to extend two results of Schatten (see [10, pp. 46-48], as well as [9, Theorems 1 and 2] for the $H^{*}$-algebra case). One extends fully, the other only partially. We use the following notation: if $\Psi$ is a linear functional on $\tau(A)$ which is continuous with respect to the norm $\tau$, we let $\tau(\Psi)$ denote the sup norm of $\Psi$ :

$$
\tau(\Psi)=\sup \{|\Psi(a)|: a \in \tau(A) \text { and } \tau(a) \leq 1\}
$$

$\tau(A)^{\prime}$ shall denote the set of all $\tau$-continuous linear functionals on $\tau(A)$. For example, $\operatorname{tr} \in \tau(A)^{\prime}$ and $\tau(\mathrm{tr})=1$.

3.1 Theorem. For $T$ in $\Lambda(A)$, define a functional $\Psi_{T}$ on $\tau(A)$ by $\Psi_{T}(a)=$ $\operatorname{tr}(T a)(a$ in $\tau(A))$. The mapping $T \rightarrow \Psi_{T}$ is a linear is ometry of $\Lambda(A)$ onto $\tau(A)^{\prime}$ : $\tau\left(\Psi_{T}\right)=\|T\|$.

Proof. See [9, Theorem 2].

Let $C^{*}(A)$ denote the $C^{*}$-algebra of $A$, the operator norm closure in $\mathscr{B}(H)$ (or in $\Lambda(A)$ ) of the space $\left\{\bar{L}_{x}: x \in A\right\}$.

3.2 Theorem. For $a$ in $\tau(A)$, define a functional $\phi_{a}$ on $C^{*}(A)$ by $\phi_{a}(T)=$ $\operatorname{tr}(T a)\left(T\right.$ in $\left.C^{*}(A)\right)$. The mapping $a \rightarrow \phi_{a}$ is a linear isometry of $\tau(A)$ into the space of continuous linear functionals on $C^{*}(A):\left\|\phi_{a}\right\|=\tau(a)$.

Proof. By Lemma 2.3, $\phi_{a}$ is a continuous linear functional on $C^{*}(A)$ and $\left\|\phi_{a}\right\| \leq \tau(a)$. The mapping $a \rightarrow \phi_{a}$ is clearly linear, so it remains only to show that $\tau(a) \leq\left\|\phi_{a}\right\|$ for each $a$ in $\tau(A)$. Use the Kaplansky density theorem (see [1, p. 46]) to obtain a sequence $\left\{z_{n}\right\}$ in $A$ with $\left\|\bar{L}_{z_{n}}\right\| \leq 1$, for all $n$, such that $W_{a}^{*}$ is the limit in the strong operator topology of $\bar{L}_{z_{n}}$. By Theorem 1.3, [a] = $\lim _{n \rightarrow \infty} z_{n} a$. Now let $\left\{e_{\gamma}: \gamma \in \Gamma\right\}$ be any projection base for $A$, and let $F$ be any finite subset of $\Gamma$. Put $p=\Sigma_{\gamma \in F} e_{\gamma}$, a projection in $A$. Since $\left\|\bar{L}_{p z_{n}}\right\| \leq$ $\left\|\bar{L}_{p}\right\|\left\|\bar{L}_{z_{n}}\right\| \leq 1$, we have

$$
\left\|\phi_{\boldsymbol{a}}\right\| \geq\left|\phi_{a}\left(\bar{L}_{p z_{n}}\right)\right|=\left|\operatorname{tr}\left(p z_{n} a\right)\right|=\left|\left(z_{n} a, p\right)\right|
$$

for each $n$. Letting $n \rightarrow \infty$, we have $\left\|\phi_{a}\right\| \geq|([a], p)|=\Sigma_{y \in F}\left([a], e_{\gamma}\right)$. Since 
$F$ is an arbitrary finite subset of $\Gamma$, this means that $\left\|\phi_{a}\right\| \geq \Sigma_{\gamma \in \mathbf{\Gamma}}\left([a], e_{\gamma}\right)=\tau(a)$, thus proving the theorem.

The difference between Theorem 3.2 and Theorem 1 if [9] is this: the image $\phi_{A}$ of the mapping $a \rightarrow \phi_{a}(a$ in $\tau(A))$ need not be all of the dual of $C^{*}(A)$. Of course this cannot be so unless $\tau$ is a complete norm on the trace-class, which means that $A$ would have to be an $H^{*}$-algebra (after trivial renorming), by Theorem 2.5. However, $\phi_{A}$ need not even be dense in the dual of $C^{*}(A)$, as the following example shows:

3.3 Example. The notation for this example is that of [3]-see especially $\$ \$ 9,10,13,19$ and 20 . Let $X$ denote an arbitrary nonvoid locally compact Hausdorff space and $\left(X, \mathbb{M}_{l}, l\right)$ a measure space of the kind discussed in $[3, \S \S 9,10]$. The measure $l$ need not be $\sigma$-finite. For convenience, put $\mathfrak{L}_{p}=\mathfrak{L}_{p}\left(x, \mathbb{M}_{l}, l\right)$, for $1 \leq p \leq \infty$. All functions considered are $\mathbb{M}_{l}$-measurable. Then $\mathfrak{L}_{2} \cap \mathfrak{L}_{\infty}$ is a commutative full Hilbert algebra under pointwise operations, the $\mathscr{L}_{2}$ inner product, and conjugation as involution. For any $f$ in $\mathfrak{L}_{2} \cap \mathfrak{L}_{\infty}, \bar{L}_{f}(g)=f g, \forall g$ in $\mathfrak{L}_{2}$. Moreover, for any $b$ in $\mathfrak{L}_{\infty}, h g \in \mathfrak{L}_{2}$ for each $g$ in $\mathfrak{L}_{2}$ and $\|b g\|_{2} \leq\|g\|_{2}\|b\|_{\infty}$. Consequently, if $b \in \mathscr{L}_{\infty}$, we shall write $\bar{L}_{b}(g)=b g, \forall g$ in $\mathfrak{L}_{2}$, noting that $\bar{L}_{b} \in \mathcal{B}\left(\mathscr{Q}_{2}\right)$ and in fact $\left\|\bar{L}_{b}\right\|=\|b\|_{\infty}$. From this, one sees that the $C^{*}$-algebra of $\mathfrak{L}_{2} \cap \mathfrak{L}_{\infty}$ is $\mathfrak{L}_{\infty}$. Using Theorem 19.30 of [3] to construct a projection base for $\mathfrak{L}_{2} \cap \mathfrak{L}_{\infty}$, one can show that $\operatorname{tr}(f)=\int_{X} f d l, \forall f$ in $\tau\left(\varrho_{2} \cap \varrho_{\infty}\right)$; thus $\tau\left(\varrho_{2} \cap \varrho_{\infty}\right)$ $=\mathscr{L}_{1} \cap \mathfrak{L}_{\infty}$ and the trace-norm is the $\mathfrak{Q}_{1}$ norm.

If the mapping $a \rightarrow \phi_{a}$ of Theorem 3.2 sent $\mathscr{L}_{1} \cap \mathfrak{L}_{\infty}$ onto a dense subset of the dual of $C^{*}\left(\mathfrak{Q}_{2} \cap \mathfrak{L}_{\infty}\right)$, then it would extend to a linear isometry $f \rightarrow \widetilde{\phi}_{f}$ of $\mathfrak{L}_{1}$ onto $\mathfrak{L}_{\infty}^{*}$ given by: $\tilde{\phi}_{f}(g)=\int_{X} f g d l, f \in \mathfrak{L}_{1}, g \in \mathfrak{L}_{\infty}$. Using the special linear isometry of $\mathcal{L}_{\infty}$ onto $\mathcal{L}_{1}^{*}$ (see $[3,19.31$ and 20.20]), one shows easily that $\mathfrak{L}_{1}$ would have to be reflexive. This is known to be false even for $X=[0,1]$ and $l$ Lebesgue measure.

4. The trace-class and two norms. The trace-class of $A$ possesses two norms, \|\| and $\tau$, neither of which is in general complete or an algebra norm. Multiplication in $\tau(A)$ is separately continuous with respect to each norm. There are two relationships between \|\| and $r$ : for any $x$ and $y$ in $A$,

$$
\tau\left(x^{*} x\right)=\|x\|^{2} \text { and } \quad \tau(x y) \leq\|x\|\|y\| \text {. }
$$

These two norms are not in general comparable-Theorem 2.5 shows that there is no constant $K$ such that $\|a\| \leq K \tau(a)$ for all $a$ in $\tau(A)$ unless $A$ is an $H^{*}$ algebra after trivial renorming, and the following result show that there is not generally any such reverse inequality. 
4.1 Theorem. The following statements are equivalent:

(1) There is a constant $K>0$ such that, for every $a$ in $\tau(A), \tau(a) \leq K\|a\|$.

(2) There is a constant $K>0$ sucb that, for every $x$ in $A,\|x\| \leq K\left\|\bar{L}_{x}\right\|$.

(3) There is a constant $M>0$ such that, for every $a$ in $\tau(A), \tau(a) \leq M \tau\left(\bar{L}_{a}\right)$.

(4) $A$ is projection bounded from above.

(5) A bas an identity.

Proof. If (1) is true then, for any $x$ in $A$,

$$
\|x\|^{2}=\tau\left(x *_{x}\right) \leq K\|x * x\| \leq K\left\|\bar{L}_{x}\right\|\|x\|
$$

so $\|x\| \leq K\left\|\bar{L}_{x}\right\|$. If (2) holds, then for any $a$ in $\tau(A)$,

$$
\tau(a)=\left\|[a]^{1 / 2}\right\|^{2} \leq K^{2}\left\|\bar{L}_{[a]^{1 / 2}}\right\|^{2}=K^{2}\left\|\bar{L}_{[a]}\right\|=K^{2}\left\|\bar{L}_{a}\right\|
$$

so (3) is true, since $\tau\left(\bar{L}_{a}\right)=\left\|\bar{L}_{a}\right\|$ by (5) of Theorem 2.4. Suppose now (3) is true; if $e$ is any projection of $A$, then $e$ is in $\tau(A)$ and $\tau(e)=\|e\|^{2}, \tau\left(\bar{L}_{e}\right)=\left\|\bar{L}_{e}\right\|=1$, therefore $\|e\| \leq M^{1 / 2}$. Thus $A$ is projection bounded from above. Suppose now there is a constant $c>0$ such that $\|p\| \leq c$ for each projection $p$ of $A$. Let $\left\{e_{y}: \gamma \in \Gamma\right\}$ be any projection base for $A$. If $F$ is any finite subset of $\Gamma$, then $\Sigma_{\gamma \in F}\left\|e_{\gamma}\right\|^{2}=\left\|\Sigma_{\gamma \in F} e_{\gamma}\right\|^{2} \leq c^{2}$, so $\Sigma_{\gamma \in \Gamma}\left\|e_{\gamma}\right\|^{2} \leq c^{2}$. Thus $\Gamma$ must be countable, so let the projection base be denoted $\left\{e_{n}\right\}_{n=1}^{\infty}$. Then $\left\{\sum_{n=1}^{m} e_{n}\right\}_{m=1}^{\infty}$ is a Cauchy sequence, so it has a limit $e=\sum_{n=1}^{\infty} e_{n}$ in $H$. Note that $e^{*}=e$. Moreover $e$ is a bounded element of $H$ : for any $y$ in $A$,

$$
L_{e}(y)=\bar{R}_{y}(e)=\lim _{m \rightarrow \infty} \bar{R}_{y}\left(\sum_{n=1}^{m} e_{n}\right)=\lim _{m \rightarrow \infty} \sum_{n=1}^{m} e_{n} y=y,
$$

since $\left\{e_{n}\right\}_{n=1}^{\infty}$ is a projection base for $A$. Therefore $e \in A$, and clearly $e y=y$ $=y e$ for all $y$ in $A$. Finally, if $A$ has an identity 1 , then for any $a$ in $\tau(A)=A$,

$$
\operatorname{tr}(a)=\operatorname{tr}(a 1)=(a, 1)
$$

(note $\left.1^{*}=1\right)$, so $\tau(a)=([a], 1) \leq\|[a]\|\|1\|=\|1\|\|a\|$. Therefore $(1)$ is true. This completes the proof of the theorem.

Yood [14, Theorem 4.2] gives other conditions on $A$ equivalent to those in the above theorem. For example, $A=C^{*}(A)$-that is, $\left\{\bar{L}_{x}: x \in A\right\}$ is closed in the operator norm topology on $\mathfrak{B}(H)$.

Having seen that these two norms on the trace-class need not be comparable, we attempt to discover what properties they have in common. To begin, we introduce some orthogonal complementation notation. If $S \subset \tau(A)$, put

$S^{+}=\{\xi \in H:(\xi, S)=\{0\}\}$, the orthogonal complement of $S$ in $H$,

$S^{\perp}=\{x$ in $A:(x, S)=\{0\}\}=S^{+} \cap A$, the orthogonal complement in $A$,

$S^{P}=\{a$ in $\tau(A):(a, S)=\{0\}\}=S^{\perp} \cap \tau(A)$, the orthogonal complement in $\tau(A)$. 
It is a consequence of Proposition 2.7 of [7] that $I^{\perp}$ is dense in $I^{+}$if $I$ is any one-sided ideal of $A$.

Much of what now follows was inspired by similar results concerning $H^{*}$-algebras in [11]. Many of our statements are formulated for left ideals only, it being understood that the corresponding results for right ideals obtain. Regarding closure terminology, a subset of $\tau(A)$ which is closed in the relative \|\| (resp. $\tau$ ) topology on $\tau(A)$ shall be referred to as " \|\| -closed" (resp. " $\tau$-closed").

4.2 Lemma. If $I$ is a left ideal of $\tau(A)$, then

(1) $T^{A}$ (the closure in $A$ of $I$ ) is a closed left ideal of $A$;

(2) $I^{P}$ is a \|\| -closed left ideal of $\tau(A)$;

(3) if $I$ is \|\|$-c l o s e d$, then $I$ is a left ideal of $A$, and $I^{P}$ is dense in $I^{\perp}$ in the norm \|\| .

Proof. (1) and (2) are easily shown using separate continuity of multiplication. If $I$ is \|\| -closed, then $I=I^{A} \cap \tau(A)$ is a left ideal of $A$. Let $x \in I^{\perp}$, and let $\left\{e_{y}: \gamma \in \Gamma\right\}$ be any projection base for $A$. Then each $e_{\gamma} x \in I^{\perp} \cap \tau(A)=$ $I^{P}$, since $\left(e_{y} x, I\right)=\left(x, e_{y} I\right)=\{0\}$. Therefore $x=\Sigma_{\gamma \in \Gamma} e_{\gamma} x \in I^{P A}$.

4.3 Lemma. If $I$ is a \|\|$-c$ losed left ideal of $\tau(A)$, then $I=A T^{A}=\{x y$ : $x \in A$ and $\left.y \in \bar{I}^{A}\right\}$.

Proof. Put $M=\left\{x y: x \in A\right.$ and $\left.y \in I^{A}\right\}$. Then $M \subset A T^{A} \subset T^{A} \cap \tau(A)=I$. Now suppose $a=x y \in I$ for some $x$ and $y$ in $A$. Since $A$ is orthocomplemented [14, Theorem 2.5] we can write $y=y_{1}+y_{2}$ with $y_{1}$ in $I^{A}$ and $y_{2}$ in $\left(I^{A}\right)^{\perp}$. By Lemma 4.2, $\left(\bar{I}^{A}\right)^{\perp}=I^{\perp}=\bar{I}^{A}$; also $\bar{I}^{A}$ and $\left(\bar{I}^{A}\right)^{\perp}$ are left ideals of $A$. Therefore $x y_{1} \in \bar{I}^{A}$ and $x y_{2} \in\left(\bar{I}^{A}\right)^{\perp}$. Hence $a-x y_{1}=x y_{2} \in \bar{I}^{A} \cap\left(\bar{I}^{A}\right)^{\perp}=(0)$, so $a=x y_{1} \in M$.

4.4 Lemma. If $\left\{e_{\gamma}: \gamma \in \Gamma\right\}$ is a projection base for $A$, then for each $a$ in $\tau(A), a=\Sigma_{\gamma \in \boldsymbol{\Gamma}} a e_{\gamma}=\Sigma_{\gamma \in \Gamma} e^{a}$ (convergence in the $\tau$ norm).

Proof. Write $a=x y$ for some $x$ and $y$ in $A$. If $F$ is any finite subset of $\Gamma$, then

$$
\tau\left(a-\sum_{\gamma \in F} a e_{\gamma}\right)=\tau\left(x y-x \sum_{\gamma \in F} y e_{\gamma}\right) \leq\|x\|\left\|y-\sum_{\gamma \in F} y e_{\gamma}\right\|
$$

This shows that $\Sigma_{\gamma \in \Gamma} a e_{\gamma}$ is summable to $a$ in the norm $\tau$. The other equality is similarly shown.

4.5 Theorem. A left ideal $I$ of $\tau(\Lambda)$ is $\tau$-closed if and only if it is \|\| -closed. 
Proof. Suppose $I$ is $\tau$-closed. We need to show that $I=I^{A} \cap \tau(A)$. If $a \epsilon \bar{I}^{A} \cap \tau(A)$, then for any $\epsilon>0$ there is a projection $p$ of $A$ such that $\tau(a-p a)<\epsilon / 2$, by Lemma 4.4. Also there is a $b \in I$ with $\|p\|\|a-b\|<\epsilon / 2$. Then $p b \in I$ and

$$
\tau(a-p b) \leq \tau(a-p a)+\tau(p a-p b) \leq \epsilon / 2+\|p\|\|a-b\|<\epsilon .
$$

Therefore $a \in I$. Hence $I$ is \|\| -closed.

Conversely, suppose $I$ is \|\| -closed. $\tau(A)$ is a dual Hilbert algebra by Theorem 2.2 and Corollary 2.2 of [14], so $I=I^{P P}$. But $I^{* P}$ is a \|\| -closed right ideal of $\tau(A)$ whose left annihilator is $I^{* P * P}=I^{P P}=I$, which is consequently $r$-closed, since the separate $\tau$-continuity of multiplication in $\tau(A)$ forces any left or right annihilator in $\tau(A)$ to be $\tau$-closed.

4.6 Corollary. The \|\|$-c l o s u r e$ of any left ideal of $\tau(A)$ is equal to its r-closure.

For the definition of an orthocomplemented Hilbert algebra, see [14, Definition 2.3]. In the proof of Theorem 4.5 we used the fact that $\tau(A)$ is a dual Hilbert algebra; it is also orthocomplemented.

4.7 Theorem. If $I$ is any closed left ideal of $\tau(A)$, then $\tau(A)=I \oplus I^{P}$.

Proof. Let $J=\bar{I}^{A}$. Then $A=J \oplus J^{\perp}$ since $A$ is orthocomplemented [14, Theorem 2.5]. For any $a \in \tau(A)$, write $a=x y$ for some $x$ and $y$ in $A$. We can write $y=y_{1}+y_{2}$ with $y_{1} \in J, y_{2} \in J^{\perp}$. Since $J^{\perp}=I^{\perp}=I^{P A}$ by Lemma 4.2 , it follows from Lemma 4.3 that $x y_{1} \in I$ and $x y_{2} \in I^{P}$. Thus $a=x y_{1}+x y_{2} \in I \oplus I^{P}$.

Thus the trace-class of a full Hilbert algebra provides another example of an orthocomplemented Hilbert algebra which is not full (see [14, Example 2.6]).

A simple argument based on the orthocomplementation property in $A$ shows that the trace-class of any closed ideal $J$ of $A$ (which is also a full Hilbert alge bra by Theorem 2.7 of [14]) is given by $\tau(J)=J \cap \tau(A)$. Using this we can obtain a characterization of the closed ideals of $\tau(A)$.

4.8 The orem. If $J$ is a closed ideal of $A$, then $r(J)$ is a closed ideal of $\tau(A)$. Conversely, any closed ideal $I$ of $\tau(A)$ bas the form $\tau(J)$ where $J=T^{A}$.

Proof. If $J$ is a closed ideal of $A$, then $\tau(J)=J \cap \tau(A)$ is clearly an ideal of $\tau(A)$, and is closed in $\tau(A)$ : the closure of $\tau(J)$ means the \|\| -closure by Corollary 4.6, so the closure of $\tau(J)$ is contained in $J \cap \tau(A)=\tau(J)$.

Suppose now that $l$ is a closed ideal of $\tau(\Lambda)$. Then $T^{A}$ is a closed ideal of $A$, so $\tau\left(I^{A}\right)=T^{A} \cap \tau(\Lambda)=I$. 


\section{BIBLIOGRA PHY}

1. J. Dixmier, Les algèbres d'opérateurs dans l'espace Hilbertien (Algèbres de von Neumann), Gauthier-Villars, Paris, 1969.

2. R. Godement, Théorie des caractères. I. Algèbres unitaires, Ann. of Math. (2) 59 (1954), 47-62. MR 15, 441 .

3. E. Hewitt and K. Stromberg, Real and abstract analysis. A modern treatment of the theory of functions of a real variable, Springer-Verlag, New York, 1965. MR 32 \#5826.

4. B. E. Johnson, An introduction to the theory of centralizers, Proc. London Math. Soc. (3) 14 (1964), 299-320. MR 28 \#2450.

5. C. N. Kellogg, Centralizers and $H^{*}$-algebras, Pacific J. Math. 17 (1966), 121-129. MR $33 \# 1749$.

6. H. Nakano, Hilbert algebras, Tôhoku Math. J. (2) 2 (1950), 4-23. MR 12, 837.

7. M. A. Rieffel, Square-integrable representations of Hilbert algebras, J. Functional Analysis 3 (1969), 265-300. MR $39 \# 6094$.

8. P. P. Saworotnow and J. C. Friedell, Trace-class for an arbitrary $H^{*}$-algebra, Proc. Amer. Math. Soc. 26 (1970), 95-100. MR $42 \# 2304$.

9. P. P. Saworotnow, Trace-class and centralizers of an $H^{*}$-algebra, Proc. Amer. Math. Soc. 26 (1970), $101-104$. MR $42 \# 2305$.

10. R. Schatten, Norm ideals of completely continuous operators, Ergebnisse der Mathematik und ihrer Grenzgebiete, N. F., Heft 27, Springer-Verlag, Berlin, 1960. MR 22 \#9878.

11. J. F. Smith, The p-classes of an $H^{*}$-algebra, Pacific J. Math. (to appear).

12. O. Takenouchi, On the maximal Hilbert algebra, Tôhoku Math. J. (2) 3 (1951), 123-131. MR 14, 290.

13. J. G. Wendel, Left centralizers and is omorphisms of group algebras, Pacific J. Math. 2 (1952), 251-261. MR 14, 246.

14. B. Yood, Hilbert algebras as topological algebras (to appear).

DEPARTMENT OF MATHEMATICS, UNIVERSITY OF OREGON, EUGENE, OREGON 97403

DE PARTMENT OF MATHEMATICS, VIRGINIA POLYTECHNIC INSTITUTE AND STATE UNIVERSITY, BLACKSBURG, VIRGINIA 24061 